\title{
More than meets the eye: The effect of planned fixations on scene representation
}

\author{
HELENE INTRAUB, JAMES E. HOFFMAN, C. JEFFREY WETHERHOLD, and STACY-ANN STOEHS \\ University of Delaware, Newark, Delaware
}

\begin{abstract}
Scene memory frequently includes a swath of unseen layout beyond a photograph's boundaries (boundary extension [BE]; Intraub \& Richardson, 1989). Might it be affected by the viewer's plan to shift fixation near a view boundary? When photographs were centrally fixated (500 msec), BE occurred following a 2-sec masked interval (Experiment 1). In Experiments 2-4, a cue during the first fixation signaled viewers to fixate an object near the left or right boundary. The picture was masked before the eyes landed. BE occurred on the cued side and on the top and bottom, but not on the uncued side. This relatively accurate performance on the uncued side suggests that inhibition of a movement to one side (in a competitive task) may also inhibit extrapolation of layout. BE on the to-be-fixated side, however, supports the idea that anticipatory representation of layout is an adaptive error that may aid the spatial integration of successive views.
\end{abstract}

The surrounding world is continuous, but sensory input is not. Viewers must sample the environment through successive movements of their eyes (and head). The first eye fixation on a natural scene is frequently sufficient to grasp its meaning and general layout (e.g., Intraub, 1981; Intraub, Gottesman, Willey, \& Zuk, 1996; Potter, 1976), but additional eye movements are required to detect specific details. Quite reasonably, the focus of most research on eye movements and memory has been to explore the impact of each new fixation on the representation of objects and details within a scene (e.g., Hollingworth \& Henderson, 2002; Irwin \& Zelinsky, 2002). The present research focuses on a different aspect of the dynamic act of visual scanning, the plan to shift fixation, and focuses on a different aspect of representation - spatial layout. The question we ask is whether the plan to fixate a new region will affect the viewer's memory for the visible expanse of the scene.

It is generally accepted that memory for a view is not like a picture in the head but is somewhat schematic in nature - a combination of general layout, gist (i.e., conceptual content), and some amount of visual detail (although exactly how much detail and how best to characterize it remains an active area of inquiry; Hochberg, 1986; Hollingworth \& Henderson, 2002; Intraub, 1997; Melcher, 2001; Rensink, 2000; Sanocki, 2003; Simons \& Levin, 1997). An interesting aspect of memory for pictures of scenes is that the representation frequently includes a specific error of commission; observers tend to remember

This research was supported by NIMH Grant MH54688 to H.I. We thank Christopher A. Dickinson, Paul Quinn, and Karen Daniels for their valuable comments on drafts of the manuscript. Correspondence should be addressed to H. Intraub, Department of Psychology, University of Delaware, Newark, DE 19716 (e-mail: intraub@udel.edu). seeing a surrounding swath of previously unseen layout just beyond the picture's edges (boundary extension [BE]; Intraub \& Richardson, 1989). BE is a striking error, occurring under conditions in which one would expect to see rather good memory-long durations (e.g., $15 \mathrm{sec}$ ) and a small number of pictures (e.g., 3-16). Viewers will confidently reject the original view, claiming it to be too close up, and will include the extended regions in pictures drawn from memory, as well as in tasks requiring reconstruction of the studied view (e.g., Gottesman \& Intraub, 2003; Intraub, 2004; Intraub \& Bodamer, 1993; for a review, see Intraub, 2002).

$\mathrm{BE}$ has been described as an adaptive memory error because it is always a good prediction of the content of the space just beyond a view boundary. In other words, although it is an error with respect to the stimulus, it captures the continuity inherent in real-world scenes and, thus, may facilitate incorporation of successive views within a larger spatial context (Intraub, 2001, 2002). It is important to note that BE occurs in memory for pictures that depict a view of the world (i.e., a scene), but not with nonscene displays (such as pictures of outline objects on blank backgrounds): The latter exhibit a bidirectional boundary error referred to as normalization (Intraub, Gottesman, \& Bills, 1998; Legault \& Standing, 1992; for other examples, see Gottesman \& Intraub, 2002, 2003). The explanation offered is that BE occurs in response to a truncated view, and the visual system treats a photograph of a scene as if it were a view that was truncated by the edges of a window. In fact, recent research has shown that memory for regions of the viewer's surrounding environment seen through a window-like aperture, are also remembered with extended boundaries (Intraub, 2004).

Because BE anticipates likely layout just beyond a visible region, there has been speculation that it might play a role in the integration of successive views during visual 
scanning (Intraub, 1997, 2002). To determine whether a brief glimpse of a scene (similar to a single fixation's worth of viewing) is sufficient to elicit BE, Intraub et al. (1996) tested BE following 250-msec presentations of photographs with multisecond stimulus onset asynchronies and following rapid serial visual presentation (RSVP) of triads of photographs (three pictures/sec). BE occurred in both cases. In the RSVP task, it occurred as soon as $1 \mathrm{sec}$ following stimulus offset. This demonstrated that it is at least plausible that BE might occur early enough to support the integration of views within a larger spatial context during visual scanning and, perhaps, might also serve to prime upcoming layout (see Sanocki, 2003, for a discussion of layout priming). Might the mere plan to fixate a region near a picture boundary influence the shape of the boundary-extended region? That is, will it affect the amount of BE that the viewer remembers beyond each of the borders?

In Experiment 1, pictures were presented for $500 \mathrm{msec}$, while the viewer maintained central fixation (monitored by an eyetracker). Presentation was followed by a 2 -sec visual noise mask, and memory was immediately tested using a new procedure (boundary adjustment) that allowed us to measure BE at each boundary independently. Using the mouse, viewers could move the black region that surrounded each test picture, thus changing each boundary so that it would reveal more or less of the scene. In Experiments 2-4, viewers fixated the center of a photograph, and then a cue directed them to "fixate an object near the left [or right] boundary"; finally, the stimulus was replaced with a mask before the eyes landed. The picture reappeared seconds later, and the viewers reconstructed the view by adjusting the boundaries. Theories of attention and memory provide at least three alternative predictions about the expected shape of the boundary-extended region under these different conditions. Each provides a different characterization of the nature of scene representation and the potential role of $\mathrm{BE}$ in view integration.

\section{THREE ALTERNATIVE HYPOTHESES}

\section{Hypothesis 1: Planned Fixations Do Not Affect Spatial Extrapolation}

Spatial extrapolation may be determined by the physical characteristics of the stimulus view. For example, wide-angle views of the same objects in a scene yield less BE than do tight close-ups (Intraub, Bender, \& Mangels, 1992; Intraub \& Berkowits, 1996). The extended region may serve as a mental map of expected space (see Hochberg, 1986) and remain unchanged until it is updated by the contents of a new fixation. If this is the case, $\mathrm{BE}$ should not be affected by the plan to fixate a new region; on average, $\mathrm{BE}$ would be expected to be no greater on the cued side of a picture than on the uncued side.

\section{Hypothesis 2: Veridical Memory on the To-Be-Fixated Side}

In contrast, scene representation may be dynamic, changing in response to shifts in attention and/or action plans (see, e.g., Rensink, 2000, for one such model). Research on eye movements and spatial attention has demonstrated that attention precedes an eye fixation and serves to improve identification of a to-be-fixated target (Deubel \& Schneider, 1996; Hoffman \& Subramaniam, 1995; Irwin \& Gordon, 1998; Kowler, Anderson, Dosher, \& Blaser, 1995: see Hoffman, 1998, for a review). Therefore, deployment of attention to a region near a boundary might be expected to result in a more veridical representation on the to-be-fixated side (no BE) than on the uncued side. This would rule out the possibility that $\mathrm{BE}$ can prime upcoming layout because, on average, the leading edge of the representation would be the one least likely to include projected layout.

\section{Hypothesis 3: More Extension on the To-Be-Fixated Side}

In contrast, research on memory for expected movement in displays raises the opposite prediction. In the case of memory for frozen-motion photographs, viewers tend to remember the object as being farther along its implied path of motion (e.g., Freyd, 1983; Futterweit \& Beilin, 1994). A similar displacement in memory occurs following the abrupt disappearance of a moving target (i.e., representational momentum; see Freyd, 1993; Hubbard, 1995; but see also Kerzel, 2000); viewers remember the target as having been slightly farther along on its path than it actually was. In an analogous way, an impending eye movement near one boundary of a view might activate anticipatory processing in that direction, resulting in an even greater amount of extrapolation on the to-be-fixated side. Thus, in direct contrast to the previous alternative, on average, $\mathrm{BE}$ would be greater on the cued side than on the uncued side.

\section{GENERAL METHOD}

All four experiments shared the same general method. Any deviation from the general method associated with an experiment will be described in the introduction to that experiment.

\section{Participants}

All the participants were male or female University of Delaware undergraduates electing to participate in the departmental participant pool. The number of participants will be reported individually for each experiment.

\section{Stimuli}

Thirty wide-angle digital photographs of natural scenes (indoor and outdoor) served as the base scenes from which stimulus views were created (Figure 1A). A computer-generated black border cropped each one so as to create stimulus views containing clearly defined objects near the right and left boundaries (Figure 1B). There were 20 pictures in Experiments 1, 2, and 4, and 30 in Experiment 3.

\section{Apparatus}

The pictures were presented on a 19-in. color monitor under the control of a Quantex 350-MHz Pentium III computer. An ISCAN table-mounted eyetracker was used to monitor eye position. The ISCAN system can detect deviations from fixation of approximately $0.25^{\circ}$ of visual angle. Samples were collected at $60 \mathrm{~Hz}$. Head position was stabilized by means of a chinrest. Stimulus views were $12.2 \times 8.5 \mathrm{~cm}$, subtending a visual angle of $9.6^{\circ} \times 6.8^{\circ}$. 
A

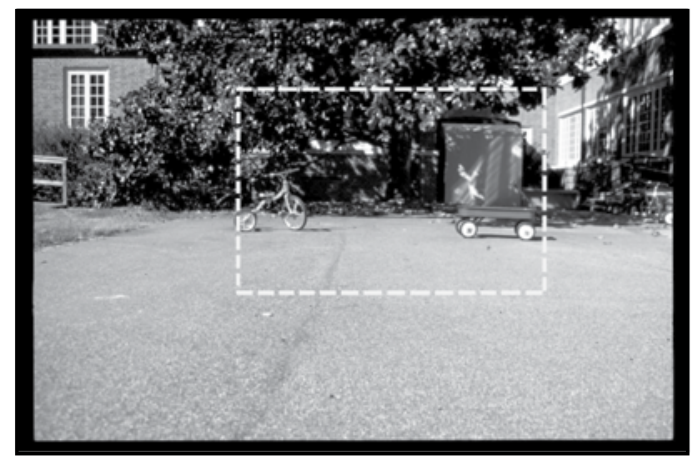

C

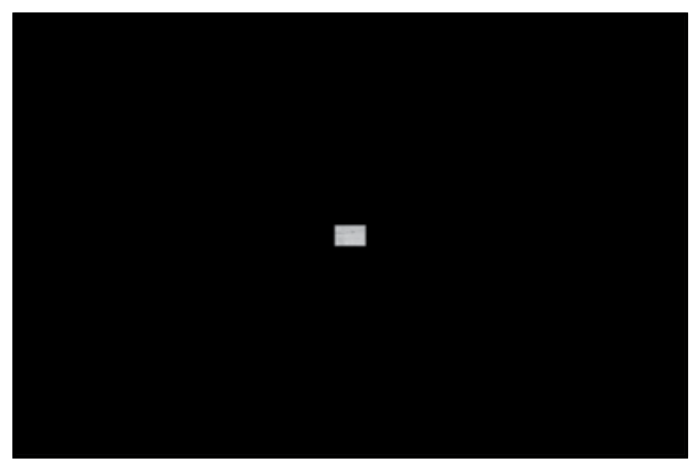

B

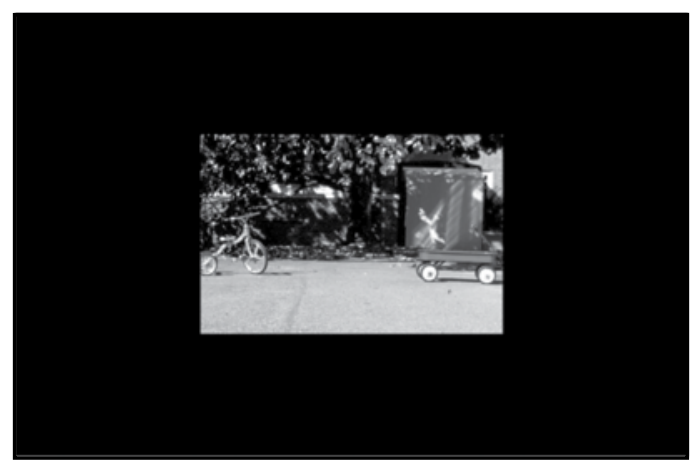

D

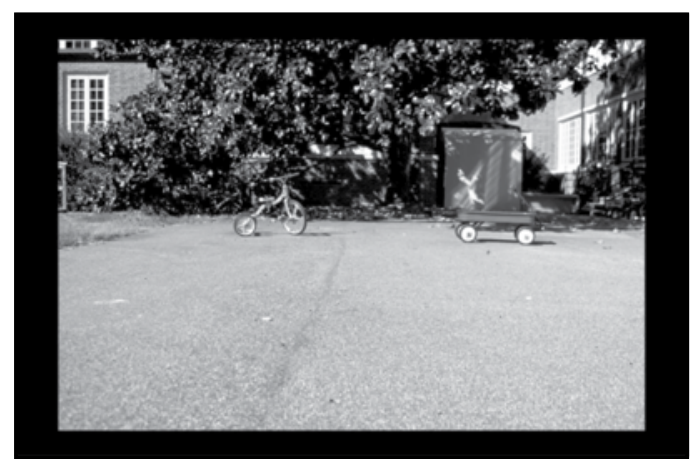

Figure 1. Base picture with dotted lines showing the stimulus view (A), the stimulus view as presented (B), and two test views: small aperture $(C)$ and large aperture $(D)$. (In the experiments, all the pictures and the mask were in color.)

\section{Procedure}

We told the participants that we were interested in their ability to voluntarily control eye movements while memorizing pictures. They were instructed to remember the objects, background, and layout when studying the stimulus and to immediately shift fixation to an object near the side designated by the cue. All the stimuli were mirror reversed for one half of the participants, to control for any biases in boundary placement that might be due to unintended differences in the left-right composition of the stimulus views; cue direction and scene orientation (normal or mirror reversed) were counterbalanced across participants. Prior to the experiment, the participants received 20 trials of practice (10 pictures not included in the experiment were presented in both normal and mirror-reversed orientations across the practice session). The trials followed the same timeline as the experimental trials, except that the memory test was excluded. Eye movement feedback was provided at the end of each practice trial (the scene reappeared with fixations superimposed and the term "correct" or "incorrect" was displayed). In the general design, eye movement feedback was limited to the practice phase of the experiment.

The timeline for each trial is shown in Figure 2. On each trial, the participant fixated a centrally located gold cross and initiated the trial when ready. The stimulus, with the fixation cross superimposed, remained on the screen for $500 \mathrm{msec}$, broken up into three periods: a precue interval $(250 \mathrm{msec})$, a cue interval ( $50 \mathrm{msec}$ during which an arrow was superimposed just above the fixation point to indicate the direction for the required eye movement), and a postcue interval $(200 \mathrm{msec})$. A visual noise mask (colorful random lines and curves on a white background) then replaced the image for $2 \mathrm{sec}$, followed immediately by the test picture, which remained on the screen until boundary adjustment was complete. To prevent bias in boundary adjustment caused by the direction in which the viewer moved the boundaries, in Experiments 1, 2, and 4, one of two types of test views were presented equally often throughout the experiment (randomly intermixed): (1) a small-aperture view, in which the boundaries were pulled in so that they almost touched (Figure 1C), and (2) a large-aperture view, in which the boundaries were pulled almost all the way out (Figure 1D). Thus, the initial test view was clearly incorrect, forcing the viewer to move the boundaries outward on small-aperture trials and inward on large-aperture trials to reconstruct the remembered view. These test views were counterbalanced across pictures. The participants then reconstructed the original view from memory, by moving the borders with the mouse. (In Experiment 3, no such movement bias existed, because the view in the test item was identical to the stimulus view.)

\section{Test Development}

In most prior $\mathrm{BE}$ recognition tests, test pictures either were identical to the stimulus or were more close-up or more wide-angle views of the same scene. Thus, the physical sizes of the stimulus and the test picture were identical; any difference reflected a change in the content of the view alone. To address the present question, however, we needed to allow the viewers to independently adjust each boundary to reveal more or less of the scene (i.e., changing not only the view, but also the size of the image with respect to the edges of the monitor). This brings up a potential artifact. Over trials, viewers might develop a strategy in which they simply adjust the size of the image with respect to the monitor, irrespective of the content of the view. In addition, they might use the outer boundaries of the test picture (by moving the borders fully outward) as a landmark that 


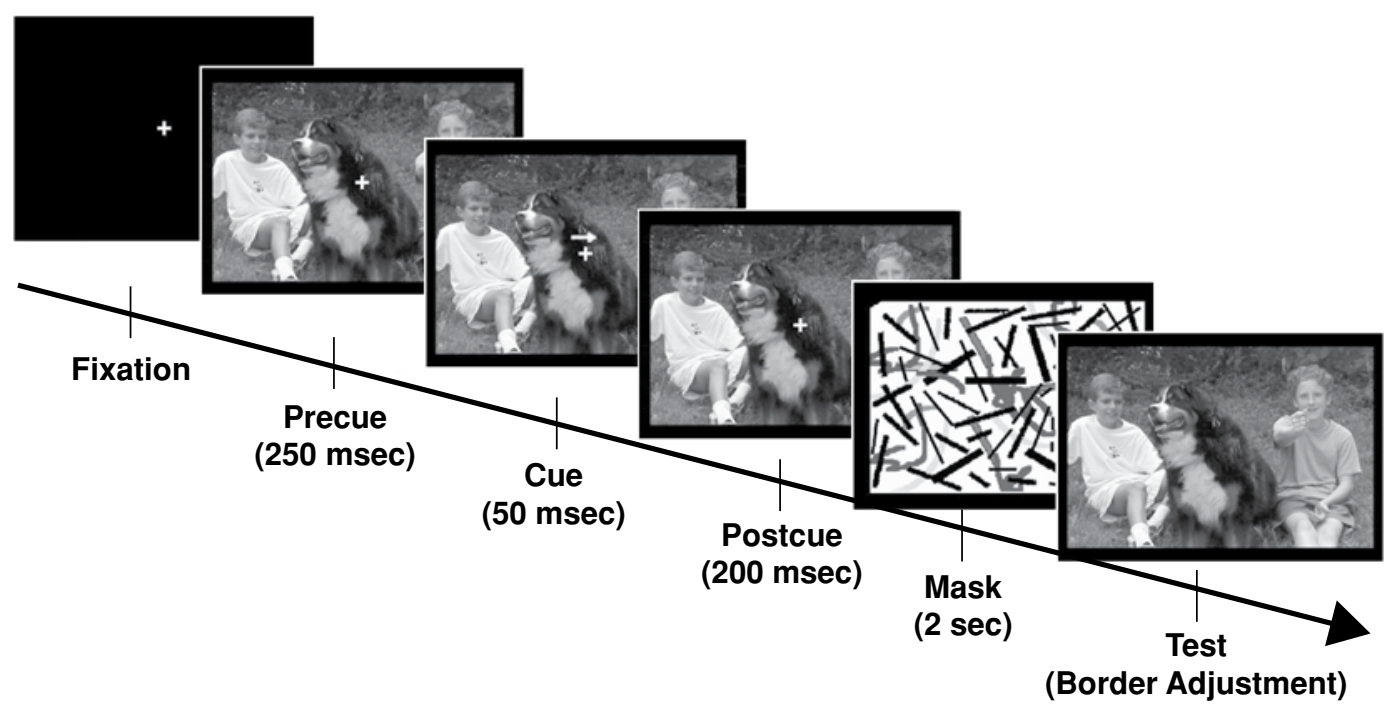

Figure 2. A sample trial is shown; timing was the same in all the experiments, but different types of test pictures were used (in this example, test item and stimulus show identical views, as in Experiment 3).

would allow them to artificially maintain the picture's aspect ratio, whether or not it was well maintained in memory. Such strategies could artificially eliminate BE. We attempted to mitigate these potential problems in several ways.

To minimize the marker problem described above, the stimulus view (which was always presented in the center of the screen) was offset with respect to the fully revealed wide-angle view (see Figure 1A). The offset differed for each picture, thus making the edges of the wide-angle view useless as a marker. As a result, if the viewer opened the boundaries to their greatest extent, the full view was not centered on the screen, although of course the portion corresponding to the stimulus view remained at center. (Note that for this reason, as well as for eliminating potential effects of individual picture compositions, a critical aspect of the design was that all the pictures were shown in both normal and mirror-reversed orientations across the experiment.) So, across the experiment, all pictures were presented in both orientations, counterbalanced with cue direction and aperture size at test. Finally, in Experiment 4, an additional variable was added. Test pictures always differed in scale from the stimulus (they were either $20 \%$ larger or $20 \%$ smaller). The rationale across all of these manipulations was to create situations in which the best strategy for the observer was to study and reproduce the content of the view, not its size or placement with respect to the monitor.

\section{Criterion Performance}

Successful trials occurred when the saccade (1) moved in the correct direction for a distance of at least $0.5^{\circ}$ from central fixation and (2) landed after the stimulus had disappeared (verified using a photocell). Task demands were very great, and the participants sometimes forgot to move their eyes or made anticipatory eye movements to an object, thus invalidating trials. An a priori criterion of at least $40 \%$ valid eye movement trials was set (distributed among counterbalancing conditions) for a participant's boundary memory to be assessed. On the basis of the robust effects in previous research, we reasoned that this number of trials would be more than sufficient to allow us to evaluate BE.

\section{Saccade Onsets (Experiments 2-4)}

In all the eye movement conditions (Experiments 2-4), saccade onset was measured from the onset of the directional cue. The me- dian onsets for saccades on valid trials in Experiments 2-4 were 360,475 , and $370 \mathrm{msec}$, respectively. These latencies are longer than those typically observed in eye movement studies in which the saccade target is indicated with a visual cue (e.g., Hoffman \& Subramaniam, 1995), but they include the time required to find a suitable object on the instructed side of a natural scene to serve as a saccade target. Note the somewhat larger latency in the three-cue condition (Experiment 3).

\section{Boundary Extension Analysis}

In all prior BE research, the questions of interest have focused on the overall area of the remembered region. To provide a comparable measurement in the present research, in each Results section, we will begin by presenting the percentage of change in area (number of pixels in the adjusted area/number of pixels in the original view). Then, to address the potential effect of planned fixations on BE, we will report the percentage of change in the distance between the center of the visible region and each boundary (i.e., the distance [in pixels] from the picture's center to an adjusted side/the distance

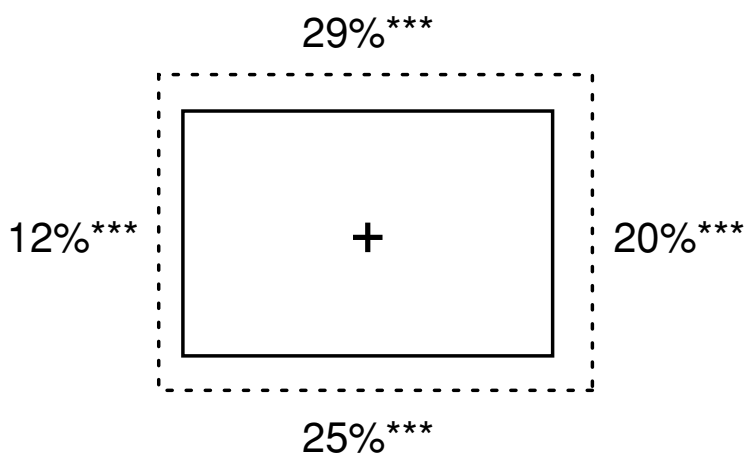

Figure 3. Mean percentage of the original distance between the picture's center and each boundary in Experiment 1 (confidence intervals were constructed around each mean). $\quad{ }^{*} p<$ $.05 .^{* * *} p<.01 .{ }^{* * *} p<.001$. 


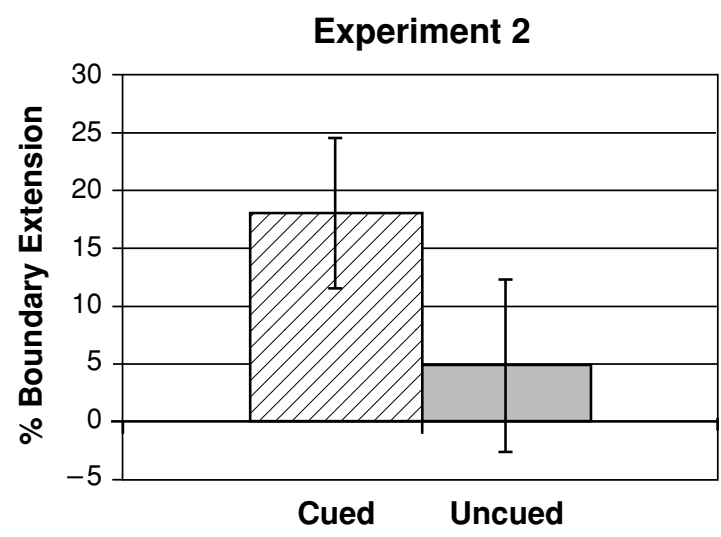

Figure 4. Mean percentages of boundary extension on the cued and uncued sides (measured from the center) in Experiment 2 (error bars show the $\mathbf{9 5 \%}$ confidence intervals).

in the original view). For both area and distance data, confidence intervals were constructed around the mean percent difference to determine whether the adjusted boundary differed significantly from the original location. Alpha was set at .05. All $t$ tests in the following analyses were two-tailed.

\section{EXPERIMENT 1}

The purpose of this experiment was to determine whether a single fixation would elicit $\mathrm{BE}$ moments later when viewers reconstructed boundaries from memory. The viewers were instructed to maintain central fixation throughout each trial. The general procedure was followed, except that the cue was a red line instead of a red arrow. This nondirectional cue served as a reminder to maintain fixation and also ensured that the sequence of events in Experiment 1 would be as similar as possible to those in the eye movement trials in the subsequent experiments. Twenty-four out of 32 participants passed criterion; on average, they maintained fixation on $80 \%$ of the trials (the range was $50 \%-100 \%$ valid trials). The 8 who did not pass criterion found it particularly difficult to suppress fixations to salient peripheral objects, given the knowledge that a reconstruction task would immediately follow.

\section{Results and Discussion}

On average, the viewers extended the boundaries, increasing the visible area by $53.7 \%(S D=41.12)$. To determine whether, on average, this reflected $\mathrm{BE}$ at each border, the change in border placement with respect to the center of the image is shown in Figure 3. As is shown in the figure, each boundary showed a significant outward shift. ${ }^{1}$ When the reconstructed areas for large- and small-aperture trials were analyzed separately, both yielded significant BE, but the distortion was greater on large-aperture trials $(75.5 \%$, $S D=51.57)$ than on small-aperture trials $(29.6 \%, S D=$ $40.25)[t(23)=5.29]$. This effect was replicated across all the subsequent experiments and will be addressed in the
General Discussion section. The new boundary adjustment procedure showed that BE occurs seconds following picture offset for a set size of one picture under conditions that make verbalizing border location (e.g., the chair is an inch from the edge) unlikely.

\section{EXPERIMENT 2}

As in Experiment 1, a new group of viewers were allowed a single fixation on each picture, but unlike in Experiment 1 , these viewers planned a fixation to an object near the right or left boundary in response to a cue (as described in the General Method section). The goal was to encourage the participants to attend to one region of space versus another. The mask replaced the picture before their eyes landed. Would the plan to fixate one side rather than the other side influence spatial memory? Specifically, would BE on the cued side differ from that on the uncued side? Twenty-two out of 26 participants passed criterion; the percentage of correct trials for these participants ranged from $40 \%$ to $95 \%$, with a mean of $63 \%$. The percentage of trials for each of the error types was the following: (1) failed to make an eye movement, $16 \%$; (2) moved in the wrong direction, $3 \%$; or (3) made anticipatory eye movements, $18 \%$.

\section{Results and Discussion}

Overall, BE again occurred. Viewers increased the visible area by $31.6 \%(S D=32.82)$, and significant increases in area occurred on both large- $(75.5 \%)$, and small$(29.6 \%)$ aperture trials $[t(23)=5.29]$. To assess the effect of planned fixations on the remembered position of individual boundaries, we collapsed across left- and right-cue conditions; the mean boundary placements for the cued side and the opposite (uncued) side are shown in Figure 4. As is shown in the figure, confidence intervals revealed a significant outward shift of the boundary on the cued side, but not on the uncued side; in addition, a $t$ test verified a significant difference in mean border placement between

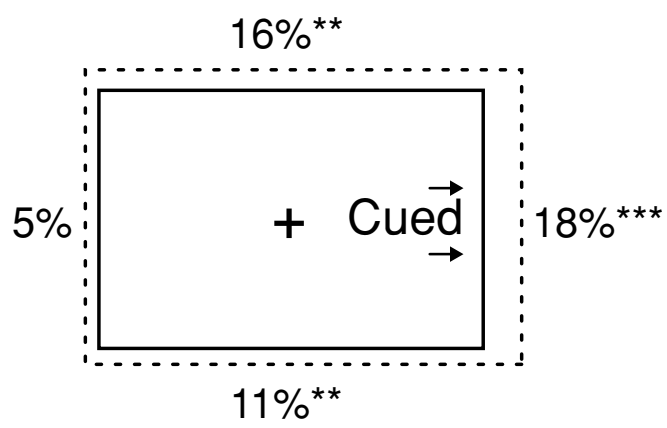

Figure 5. Mean percentages of the original distance between the picture's center and the top, bottom, and cued and uncued sides, collapsing over left and right cues (confidence intervals were constructed around each mean). $\quad{ }^{*} p<.05 .{ }^{* *} p<.01 .{ }^{* * *} p<$ .001 . 


\section{Experiment 2: Left vs. Right}

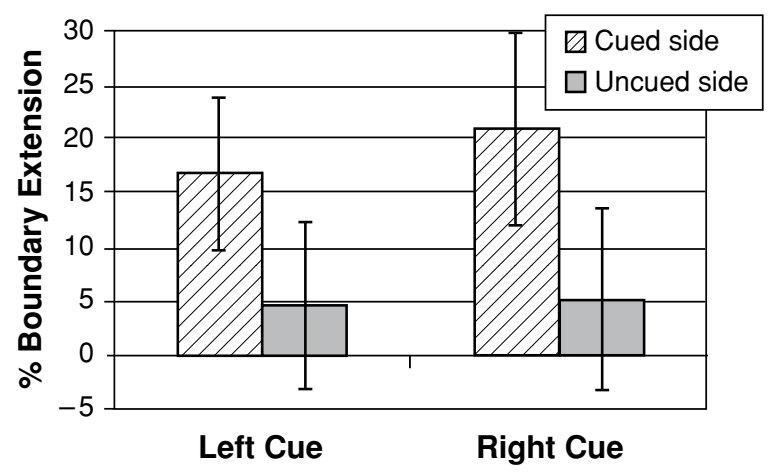

Figure 6. Mean percentages of boundary extension on the cued and uncued sides (measured from the center) for right-cue and left-cue trials in Experiment 2 (error bars show the 95\% confidence intervals).

the cued and the uncued sides $[t(21)=3.22]$. Figure 5 shows mean boundary placement for all four boundaries; $\mathrm{BE}$ occurred not only on the cued side, but also on the top and bottom.

These results run contrary to both Hypotheses 1 and 2, in that the plan to shift fixation did influence spatial memory but did not do so by eliminating BE on the to-be-fixated side. Was BE enhanced on the cued sides (Hypothesis 3)? We directly compared $\mathrm{BE}$ on the cued sides $(18.0 \%, S D=$ 14.6) with boundary placement at the sides (collapsing over left and right) in Experiment $1(16.2 \%, S D=13.38)$; performance did not differ $[t(44)<1]$. The plan to fixate neither decreased nor increased $\mathrm{BE}$ on the cued side but, apparently, eliminated BE on the uncued side.

Left cues versus right cues. The same pattern of results for cued and uncued sides was observed when leftcue and right-cue trials were analyzed separately. Although power was greatly reduced, as is shown in the Figure 6, confidence intervals revealed significant outward shifts of the boundary on the cued side, but not on the uncued side, on both left- and right-cue trials. A significant difference between cued and uncued sides was again obtained, irrespective of cue direction $[t(21)=2.10$ (left-cue trials) and $t(21)=2.08$ (right-cue trials)]. Analysis of the top and bottom borders also followed the same pattern as the overall analysis, although with reduced power; the leftcue-bottom displacement failed to reach significance.

The results of Experiment 2 suggest that the plan to fixate near a boundary does not eliminate $\mathrm{BE}$ on the tobe-fixated side. In fact, the amount of BE did not differ from that obtained when viewers simply maintained fixation (Experiment 1). However, the results did reveal an unexpected outcome: elimination of BE on the side opposite the planned fixation. Somewhat counterintuitively, the "rejected" side yielded, on average, a more accurate assessment of true boundary placement. This suggests another alternative that was not considered at the outset of the study: inhibition, as discussed in the biased competi- tion model of attention (Desimone, 1998; Desimone \& Duncan, 1995; see the General Discussion section). Perhaps, in this go/no-go situation, inhibiting a movement to the uncued side also served to inhibit the development of $\mathrm{BE}$ on that side. If replicated, this would raise a new consideration about scene representation during the dynamics of visual scanning.

\section{EXPERIMENT 3}

The purpose of Experiment 3 was to determine whether this unexpectedly accurate performance on the side opposite the cue could be replicated under other conditions. In Experiment 1, the viewers were consistently cued to maintain central fixation; there were no competing cues to monitor. In contrast, in Experiment 2, the shift fixation conditions forced the viewers to monitor competing cues (i.e., look left vs. look right) while studying the picture. In Experiment 3, we implemented a three-cue task in which left and right cues (red arrows) were randomly interspersed with maintain central fixation cues (red circle around the fixation point). In this way, we attempted a replication of Experiment 2 while, at the same time, exploring the impact of cue competition on trials requiring the viewer to maintain central fixation. Following the logic of biased competition on no-movement trials (circle cue), suppression of both competing actions should occur, resulting in elimination of BE on both sides, but not on the top and bottom.

In pilot tests, we found that the addition of the third cue made what was already a difficult task far too difficult for most viewers (barely one third of the viewers passed criterion). The demands of monitoring three cues superimposed on natural scenes, followed by a demanding memory test, were simply too great. Therefore, two changes were made to the general method: (1) Eye movement feedback identical to that provided during practice in the previous experiments was presented at the end of each experimental trial (the full view of the scene appeared, with fixations super-

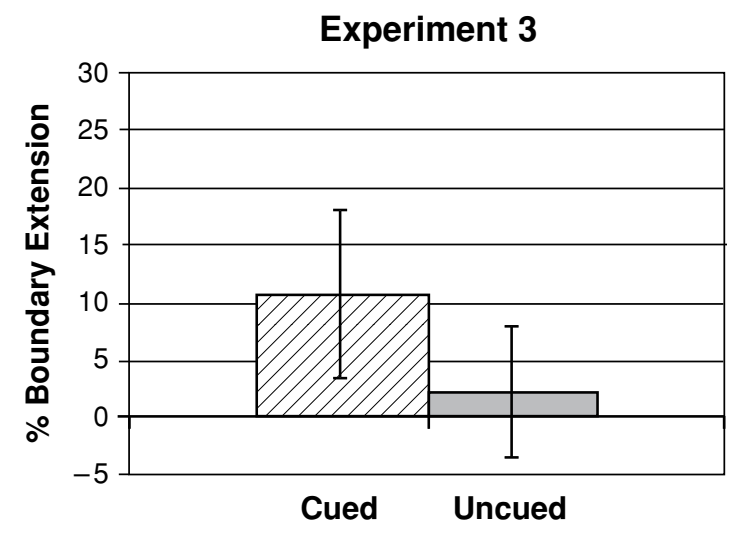

Figure 7. Mean percentages of boundary extension on the cued and uncued sides (measured from the center) in Experiment 3 (error bars show the $\mathbf{9 5 \%}$ confidence intervals). 


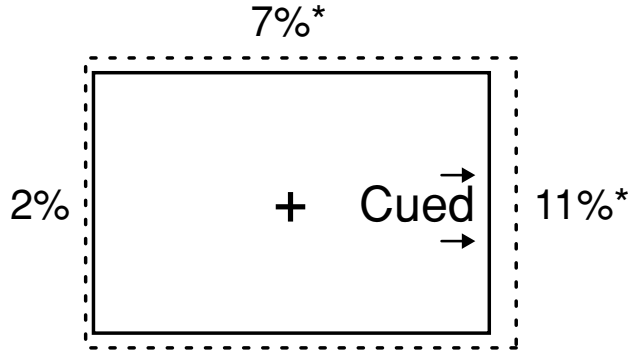

$8 \%$ *

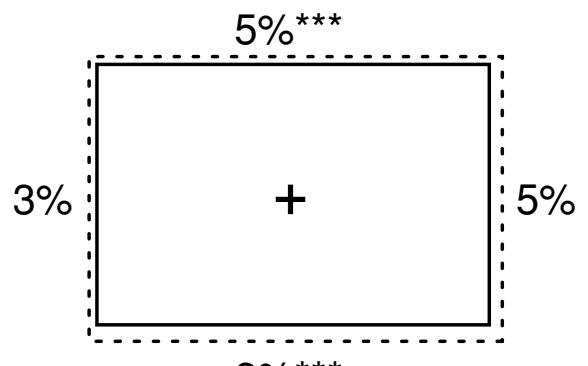

$8 \%{ }^{* * *}$

Figure 8. Top panel shows the mean percentage of the original distance between the picture's center and the top, bottom, and uncued and cued boundaries, collapsing over left- and right-cue trials. The bottom panel shows the same for trials on which the participants were cued to maintain fixation at the center in $\mathbf{E x}$ periment 3 (confidence intervals were constructed around each mean). $\quad{ }^{*} p<.05 . \quad{ }^{* *} p<.01 . \quad{ }^{* * *} p<.001$.

imposed and the term "correct" or "incorrect" displayed), and (2) the memory test was simplified by eliminating the aperture variable. With these changes, 39 out of 42 participants passed criterion; the percentage of valid trials for these participants ranged from $43 \%$ to $97 \%$, with a mean of $55 \%$. The percentage of trials for each of the

\section{Experiment 3: Left vs. Right}

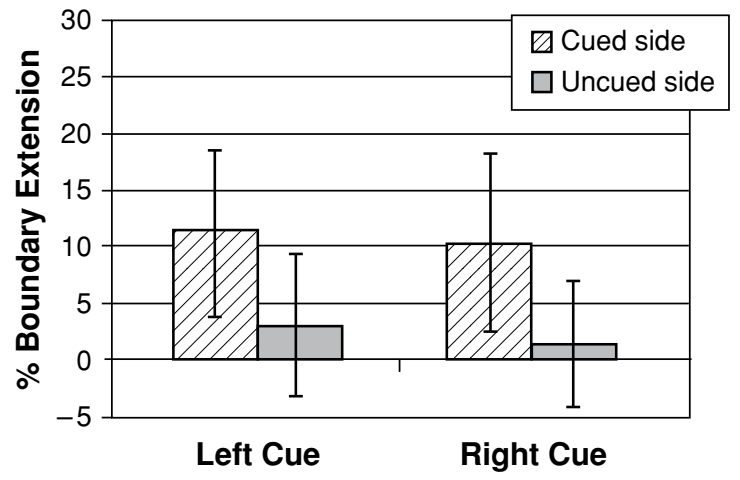

Figure 9. Mean percentages of boundary extension on the cued and uncued sides (measured from the center) for right-cue and left-cue trials in Experiment 3 (error bars show the 95\% confidence intervals). error types was the following: (1) failed to make an eye movement, $22 \%$; (2) moved in the wrong direction, $1 \%$; or (3) made anticipatory eye movements, $8 \%$. On trials requiring the participants to maintain fixation, they moved their eyes on $20 \%$ of the trials (similar to the error rate in Experiment 1).

Thus, as is illustrated in Figure 2, boundary placement in the picture was identical across the entire trial (stimulus, mask, and test), thus providing an extremely conservative test of BE.

\section{Results}

Eye movement trials (arrow cues). The viewers remembered seeing beyond the boundaries, increasing the visible area by $18.3 \%(S D=48.31)$. Boundary placement on the cued and uncued sides is shown in Figure 7. Once again, the viewers shifted the cued side outward, but not the uncued side, and mean distance from the center of the image differed between the two $[t(38)=3.21]$. Figure 8 (upper panel) shows performance at all four boundaries on eye movement trials. Again, all the sides yielded BE except the side opposite the cue.

No movement trials (circle cues). On these trials, to maintain central fixation, the viewers had to refrain from making both leftward and rightward eye movements. Figure 8 (lower panel) shows that consistent with the biased competition prediction, no BE was obtained on the sides, but significant BE did occur at the top and bottom boundaries. Simply put, when the cues were placed in conflict, the never-cued top and bottom always yielded BE. On eye movement trials (arrow cues), BE occurred on the cued side, but not on the uncued side (i.e., the "path not taken" in the two-way competition); whereas on no-movement trials (circle cue), which required inhibition of both competing actions, BE occurred on neither side (i.e., the "paths not taken").

Left cues versus right cues. Finally, as in the previous experiments, to determine whether the same pattern occurred on left-cue and right-cue trials, we analyzed them separately. As is shown in Figure 9, BE occurred on the cued side, but not on the uncued side, irrespective of cue direction. In both cases, $t$ tests showed that there was a significant difference in performance between the cued and the uncued sides $[t(38)=2.80$ (left cues) and $t(38)=$ 2.02 (right cues)]. The top and bottom borders showed a pattern similar to that for the combined results; all but the right-cue-bottom displacement reached significance.

\section{EXPERIMENT 4}

Can we be sure that the new border adjustment task is really demonstrating BE? Instead, could the results be explained by assuming that the viewers might have been expanding the size of the aperture to fit a preferred size with respect to the screen? Because the full image and the stimulus view (Figure 1, panels A and B, respectively) were always held constant across presentation and test in Experi- 


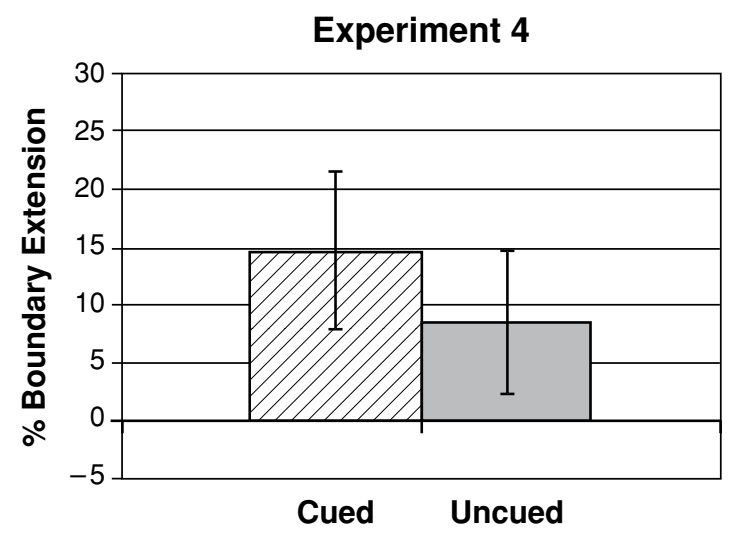

Figure 10. Mean percentages of boundary extension on the cued and uncued sides (measured from the center) in Experiment 4 (error bars show the $95 \%$ confidence intervals).

ments $1-3$, one cannot tell. To determine whether the viewers were simply adjusting the aperture to create a preferred image size with respect to the screen (instead of remembering a greater expanse of the scene being shown in the stimulus view), in Experiment 4, we replicated Experiment 2 but did so with the addition of a new test variable - scale.

The stimulus view was always the same as that in Experiment 2 , but the test image always differed in terms of scale; it was either compressed ( $20 \%$ smaller) or expanded (20\% larger). The difference was very noticeable, and the viewers were forewarned of the size change that would occur. They were told to reconstruct the view that they had just studied, ignoring the size of the test picture. Scale and aperture size were both varied, making an already difficult task (Experiment 2) extremely difficult. Although a total of 57 participants had to be run to obtain 33 who could pass criterion, we felt it worthwhile to determine whether those who were able to accomplish the task would yield $\mathrm{BE}$ results similar to those in Experiment 2, thus support-

\section{Experiment 4: Left vs. Right}

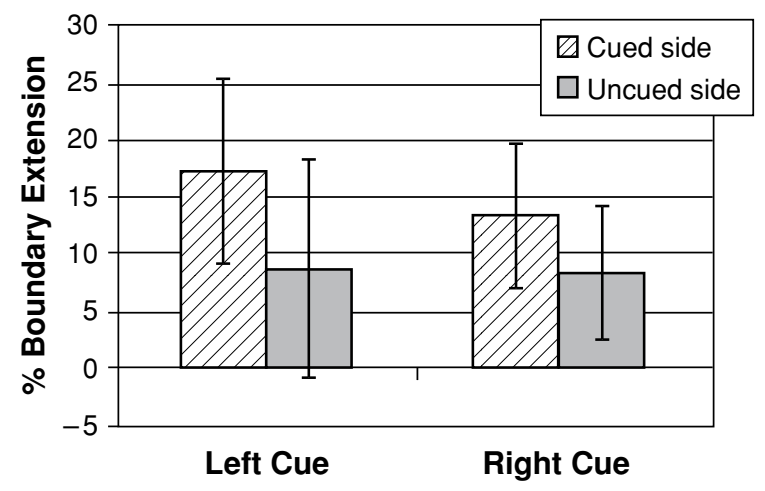

Figure 11. Mean percentages of boundary extension on the cued and uncued sides (measured from the center) for right-cue and left-cue trials in Experiment 4 (error bars show the 95\% confidence intervals). ing the idea that the viewers were misremembering the expanse of the view (i.e., true BE), or whether their border adjustments would reveal that the viewers were actually reconstructing a preferred stimulus size with respect to the screen. The percentage of valid trials for those passing criterion ranged from $40 \%$ to $95 \%$, with a mean of $63 \%$. The percentage of trials for each of the error types was the following: (1) failed to make an eye movement, 17\%; (2) moved in the wrong direction, $3 \%$; or (3) made anticipatory eye movements, $17 \%$.

\section{Results}

Overall BE. Although the scale of the test image always differed from that of the stimulus view, the overall $\mathrm{BE}$ was similar to that obtained in Experiment 2; viewers remembered having seen $35.7 \%$ more of the scene than had been shown. Significant BE was obtained for smallscale views $(15.5 \%, S D=29.9)$ and for large-scale views $(58.4 \%, S D=52.1)$. As in the previous experiments, significant amounts of $\mathrm{BE}$ occurred on both large-aperture and small-aperture trials ( $50.2 \%$ and $23.7 \%$, respectively), with large-aperture trials yielding more $[t(32)=4.23]$.

Cued versus uncued sides: Overall, and left cue versus right cue. Figure 10 shows that performance on the cued and uncued sides was generally similar to that obtained in Experiments 2 and 3. The only difference was that $\mathrm{BE}$ was reduced on the uncued side, relative to the cued side $[t(32)=2.30]$, rather than eliminated. Figure 11 shows that this was due to performance in the right-cue condition. For left cues, BE occurred on the cued side, but not on the uncued side, and performance differed between the two $[t(32)=2.10]$. For right cues, significant BE occurred for both sides; the pattern reflected more BE on the cued side, but this difference did not reach significance $[t(32)=1.67, p<.10]$.

\section{GENERAL DISCUSSION}

A single fixation was sufficient to elicit boundary extension when memory was tested moments following presentation (Experiments 1-4). With a new quantitative method for testing BE (boundary adjustment), viewers demonstrated memory beyond the given view whether the test picture showed more of the scene than had been presented in the stimulus view or less (Experiments 1, 2, and $4)$. Boundary adjustments revealed BE even when the test view was identical to the stimulus (Experiment 3 ). Clearly, these overexpansive errors in memory were related to the content of the scene and were not simply the remembered size of the image, because BE occurred both when the test image was expanded $(20 \%$ larger in size than the stimulus) and when it was compressed $(20 \%$ smaller in size than the stimulus) in Experiment 4. The viewers simply remembered having seen more of the scene than had been physically shown, moments after a single fixation.

Did the plan to shift fixation toward one side of the stimulus view improve spatial memory on that side, thus eliminating BE on that side? The results of Experiments 2-4 showed that when a fixation was planned to- 
ward a region near the left or right boundary, BE was not eliminated on the to-be-fixated side. This shows that the obligatory shift in spatial attention that precedes a fixation (e.g., Hoffman \& Subramaniam, 1995; Kowler et al., 1995) does not eliminate BE in an attended region. A comparison of BE on the to-be-fixated side (Experiment 2) with that obtained on the sides when the viewers were always required to maintain central fixation (Experiment 1) revealed no evidence that an imminent fixation had any effect on the remembered space on the to-be-fixated side. In other words, BE appears to be available at the leading edge of the representation during the dynamic act of visual scanning.

An unexpected outcome of the large- and small-aperture conditions (Experiments 1, 2, and 4) may provide new insight into the nature of the representation available during view integration. Although mean remembered area was overinclusive on both types of trials, extrapolation always was greater in the case of the large apertures. Why might this bias occur? Consider the possibility that the representation of a view, like the retina that originally registered the ambient array, is inhomogeneous. If the representation (which most researchers agree is more schematic than photographic) becomes less detailed (less substantial) the farther into the periphery one tests, a large aperture, which shows the viewer the layout beyond the original boundaries at the time of the test, might cause even "wispy" peripheral regions of the representation to pass threshold for recognition during boundary adjustment, whereas a small test aperture, which does not even show the original boundaries, might not. It is critical to remember that BE still occurred both in the small-aperture conditions and in a condition in which the test view was identical to the stimulus view (Experiment 3). This is consistent with prior research on boundary extension showing that the region immediately beyond a boundary was so much a part of the viewer's representation that even foreknowledge about BE and attempts to overcome it failed (e.g., Intraub \& Bodamer, 1993; Intraub et al., 1996). The large aperture simply serves to enhance this anticipatory representation of surrounding space.

The plan to fixate near a boundary did not eliminate BE on that side, but did it affect the shape of the boundaryextended region in some other way? All the viewers were allowed the same $200 \mathrm{msec}$ of inspection time prior to the onset of the cue, yet the action plan that emerged on the basis of the cue had an influence on memory. The plan to fixate in one direction affected memory on the side opposite the planned fixation; somewhat surprisingly, the uncued, "rejected" side provided, on average, the best reproduction of border placement of the four. BE was actually eliminated (Experiments 2 and 3 ) or reduced (Experiment 4) on the uncued side. The results of the eye movement experiments (Experiments 2-4) show that BE is not determined solely by stimulus characteristics (e.g., how close-up or wide-angle the view is; Intraub et al., 1992); action plans appear to have mattered. But how can one explain why border placement was apparently more accurate on the side opposite the planned fixation - that is, why it failed to show a directional bias?

Perhaps, rather than an improvement in memory, the observation reflects a shift of the entire representation (maintaining its aspect ratio) in the direction of the eye movement. A comparison of BE between Experiments 1 and 2 suggests that this is not the case, because BE on the cued side in Experiment 2 did not differ from that obtained on the sides in Experiment 1, in which viewers always maintained central fixation. Also, unlike Experiment 1, the no-movement condition in Experiment 3 resulted in $\mathrm{BE}$ at the top and bottom, but not at the sides. An alternative explanation that is consistent with the pattern of results across experiments is that inhibition of a competing action (i.e., movement to the uncued side) also resulted in inhibition of spatial extrapolation on that side of the image. This possibility is consistent with the concepts expressed in the biased competition model of attention (Desimone, 1998; Desimone \& Duncan, 1995). Central to this neural model of attention is the claim that selective attention does not simply enhance neuronal firing to an attended target but that it inhibits competing inputs.

For example, reduced neural firing rates were observed in the macaque inferior temporal cortex for cells responding to an uncued object prior to a saccade, in comparison with the cued object (Chelazzi, Duncan, Miller, \& Desimone, 1998; Chelazzi, Miller, Duncan, \& Desimone, 2001), and similar effects have been observed in other areas of the visual cortex (e.g., Moran \& Desimone, 1985; see Awh, Matsukura, \& Serences, 2003, for a behavioral analogue with humans). We are, of course, fully aware that these results focus on attention to objects in space and that we are addressing memory for a top-down generated background. However, there is no in principle reason that the model cannot be extended to account for selection of areas or regions, as well as objects. Applied to the present research, inhibition of the processes associated with the uncued region might suppress the processes that normally elicit BE. This account would be consistent with the observation that BE occurred not only on the cued side, but also on the irrelevant top and bottom boundaries; it was the uncued side that tended to be free of BE (or as in Experiment 4, in which both the pictures scale and the aperture size changed at test, the uncued side yielded relatively little $\mathrm{BE}$ ).

In the present experiments, there were always two peripheral objects competing for attention, and the task specifically enhanced competition by the addition of the cue. The viewer never knew in advance whether the cue would be to the left or to the right. Still, must we appeal to inhibition to explain these results? Alternatively, consider the possibility that BE occurs only at an attended location and, because the cue drew attention away from the uncued side, BE simply was not "turned on." This is unlikely because, as was noted earlier, the top and bottom boundaries were never the targets of a cue, yet they always yielded BE (Experiments 1-4). The inhibition explanation, on the other hand, is consistent with prior evidence suggesting 
that $\mathrm{BE}$ is a default process in scene representation (Gottesman \& Intraub, 2002; Intraub \& Bodamer, 1993) that may have been actively suppressed on the rejected sideon the "path not taken" in a competitive context.

A comparison of the maintain central fixation conditions in Experiments 1 and 3 suggests additional support for this possibility. The viewers in Experiment 1 were not burdened with monitoring competing cues and launching saccades while they studied the stimulus. On the face of it, one would expect the most veridical boundary memory in that condition; yet, if anything, that experiment yielded more BE than was shown in any of the other experiments, and extrapolation occurred at all four boundaries. As was mentioned earlier, in contrast, when the viewers maintained central fixation in the context of a three-way competition (left vs. right arrow vs. circle cue in Experiment 3), BE occurred at the top and bottom, but not on the sides. This is important because both of the sides in this case were simultaneously the "paths not taken."

The lack of BE on the sides in this case cannot be attributed to the simplified memory test in Experiment 3 (test boundaries were identical to stimulus boundaries) because, when the cue elicited an eye movement (left or right cue) in the same experiment, BE occurred not only on the top and bottom, but on the cued side as well. Only when a side was a "path not taken" did BE fail to occur. Whether similar patterns of BE would be obtained during free viewing of the stimuli used in these experiments is a topic for future research. At issue is whether the competition induced by the presence of salient objects on both sides of the view would be sufficient to elicit inhibition, or whether it requires the heightened competition of moving the eyes in response to competing cues.

The unanticipated inhibitory effects observed in these experiments are reminiscent of findings reported by Tipper, Howard, and Houghton (1998) in the context of action-based mechanisms of attention. They had their participants reach toward targets that were surrounded by distractors and found that interference from the distractors was a function of their distance from the responding hand. In addition, they reported that reaching toward the ignored distractor on a subsequent trial was slowed, suggesting that ignoring the distractor was accomplished by an inhibitory process that lingered long enough to affect subsequent reaches. In the present experiments, viewers may have actively inhibited processing of the potential target on the uncued side, which, in turn, reduced the otherwise automatic elicitation of projected layout.

Does the biased competition explanation fly in the face of what is currently known about attention and memory? The way in which visual attention is distributed around an attended location has been a subject of vigorous debate. The distribution of attention has sometimes been likened to a spotlight (Eriksen \& Hoffman, 1973) or a variable power zoom lens (Eriksen \& St. James, 1986), in which enhanced processing is centered on the attended location. However, evidence has also appeared for a inhibitory area surrounding the attended location (Bahcall \& Kowler,
1999), as well as for enhanced processing of locations diametrically opposite the attended one (Tse, Sheinberg, $\&$ Logothetis, 2003). We currently do not know what factors contribute to these different spatial allocations of attention, but at this stage, we certainly cannot rule out selective inhibition of unattended areas, particularly given that BE might rely on a different attentional deployment than that used for perceiving or remembering details of an attended target. For example, inhibition of early visual areas on the uncued side might carry forward to higher level brain areas (a highly speculative candidate would be the parahippocampal place area; Epstein \& Kanwisher, 1998 - see also Intraub 2002) associated with scene perception and layout representation, thus minimizing BE on the uncued side.

In conclusion, whether or not inhibition turns out to be the best explanation of the relatively "good" spatial memory on the uncued side, the most important outcome of the present studies are that a robust BE error repeatedly occurred on the cued side-what would be the leading edge of the representation if one were allowed to complete the fixation and see the world beyond the view. It is well known that the amount of BE is affected by stimulus characteristics (e.g., how close-up a view is [Intraub et al., 1992]; and, perhaps, the emotional content of the picture [Mathews \& Mackintosh, 2004]). The present research demonstrates that the amount and shape of this extended region is also affected by the direction of an upcoming fixation.

The results suggest as well that the mental representation of a view may be inhomogeneous in a manner similar to the visual field itself, with peripheral areas represented less well than central areas. This could serve an adaptive value if, as has been discussed elsewhere (Intraub, 2001, 2002 ), the visual system has evolved to ignore the spurious boundaries of a given view and extrapolate beyond them - thus capturing the continuity of real-world scenes. The outcome of the present experiments supports the idea that boundary extension is a default process of layout projection that occurs rapidly, given a single fixation on a picture. Its presence so soon after stimulus offset underscores the possibility that this anticipatory spatial representation may play a role in the viewer's ability to understand and coherently represent a surrounding world that can be perceived only a part at a time.

\section{REFERENCES}

Awh, E., Matsukura, M., \& Serences, J. T. (2003). Top-down control over biased competition during covert spatial orienting. Journal of Experimental Psychology: Human Perception \& Performance, 29, 52-63.

BAHCALl, D. O., \& Kowler, E. (1999). Attentional interference at small spatial separations. Vision Research, 39, 71-86.

Chelazzi, L., Duncan, J., Miller, E. K., \& Desimone, R. (1998). Responses of neurons in inferior temporal cortex during memory-guided visual search. Journal of Neurophysiology, 80, 2918-2940.

Chelazzi, L., Miller, E. K., Duncan, J., \& Desimone, R. (2001).

Responses of neurons in macaque area V4 during memory-guided visual search. Cerebral Cortex, 11, 761-772.

Desimone, R. (1998). Visual attention mediated by biased competition 
in extrastriate visual cortex. Philosophical Transactions of the Royal Society of London: Series B, 353, 1245-1255.

Desimone, R., \& Duncan, J. (1995). Neural mechanisms of selective visual attention. Annual Review of Neuroscience, 18, 193-222.

Deubel, H., \& Schneider, W. X. (1996). Saccade target selection and object recognition: Evidence for a common attentional mechanism. Vision Research, 36, 1827-1837.

EPSTEIN, R., \& KANWISHER, N. (1998). A cortical representation of the local visual environment. Nature, 392, 598-601.

Eriksen, C. W., \& Hoffman, J. E. (1973). The extent of processing of noise elements during selective encoding from visual displays. Perception \& Psychophysics, 14, 155-160.

Eriksen, C. W., \& St. James, J. D. (1986). Visual attention within and around the field of focal attention: A zoom lens model. Perception \& Psychophysics, 40, 225-240.

FREYD, J. J. (1983). The mental representation of movement when static stimuli are viewed. Perception \& Psychophysics, 33, 575-581.

FREYD, J. J. (1993). Five hunches about perceptual processes and dynamic representations. In D. E. Meyer \& S. Kornblum (Eds.), Attention and performance XIV: Synergies in experimental psychology, artificial intelligence, and cognitive neuroscience (pp. 99-119). Cambridge, MA: MIT Press.

Futterweit, L. R., \& Beilin, H. (1994). Recognition memory for movement in photographs: A developmental study. Journal of Experimental Child Psychology, 57, 163-179.

Gottesman, C. V., \& Intraub, H. (2002). Surface construal and the mental representation of scenes. Journal of Experimental Psychology: Human Perception \& Performance, 28, 1-11.

Gottesman, C. V., \& Intraub, H. (2003). Constraints on spatial extrapolation in the mental representation of scenes: View-boundaries versus object-boundaries. Visual Cognition, 10, 875-893.

Halpern, A. R., \& Kelly, M. H. (1993). Memory biases in left versus right implied motion. Journal of Experimental Psychology: Learning, Memory, \& Cognition, 19, 471-484.

HochBerG, J. (1986). Representation of motion and space in video and cinematic displays. In K. R. Boff, L. Kaufman, \& J. P. Thomas (Eds.), Handbook of perception and human performance (Vol. 1, pp. 22:122:64). New York: Wiley.

Hoffman, J. E. (1998). Visual attention and eye movements. In H. Pashler (Ed.), Attention (pp. 119-154). London: University College London Press.

Hoffman, J. E., \& Subramaniam, B. (1995). The role of visual attention in saccadic eye movements. Perception \& Psychophysics, 57, 787-795.

Hollingworth, A., \& Henderson, J. M. (2002). Accurate visual memory for previously attended objects in natural scenes. Journal of Experimental Psychology: Human Perception \& Performance, 28, $113-136$.

HubBaRd, T. L. (1995). Environmental invariants in the representation of motion: Implied dynamics and representational momentum, gravity, friction, and centripetal force. Psychonomic Bulletin \& Review, 2, 322-338.

INTRAUB, H. (1981). Rapid conceptual identification of sequentially presented pictures. Journal of Experimental Psychology: Human Perception \& Performance, 7, 604-610.

InTRAub, H. (1997). The representation of visual scenes. Trends in Cognitive Sciences, 1, 217-221.

INTRAUB, H. (2001). Internalized constraints in the representation of spatial layout. Behavioral \& Brain Sciences, 24, 677-678.

InTRAUB, H. (2002). Anticipatory spatial representation of natural scenes: Momentum without movement? Visual Cognition, 9, 93-119.

IntRaub, H. (2004). Anticipatory spatial representation in a deaf and blind observer. Cognition, 94, 19-37.

Intraub, H., Bender, R. S., \& Mangels, J. A. (1992). Looking at pictures but remembering scenes. Journal of Experimental Psychology: Learning, Memory, \& Cognition, 18, 180-191.

IntRAUB, H., \& Berkowits, D. (1996). Beyond the edges of a picture American Journal of Psychology, 109, 581-598.

IntRaUb, H., \& BoDAmer, J. L. (1993). Boundary extension: Fundamental aspect of pictorial representation or encoding artifact? Journal of Experimental Psychology: Learning, Memory, \& Cognition, 19 , 1387-1397.

Intraub, H., Gottesman, C. V., \& Bills, A. J. (1998). Effects of perceiving and imagining scenes on memory for pictures. Journal of Experimental Psychology: Learning, Memory, \& Cognition, 24, 186-201.

Intraub, H., Gottesman, C. V., Willey, E. V., \& Zuk, I. J. (1996). Boundary extension for briefly glimpsed photographs: Do common perceptual processes result in unexpected memory distortions? Journal of Memory \& Language, 35, 118-134.

InTRAUB, H., \& RichaRDSON, M. (1989). Wide-angle memories of closeup scenes. Journal of Experimental Psychology: Learning, Memory, \& Cognition, 15, 179-187.

IrWIN, D. E., \& GoRDON, R. D. (1998). Eye movements, attention and trans-saccadic memory. Visual Cognition, 5, 127-155.

Irwin, D. E., \& ZelinsKy, G. J. (2002). Eye movements and scene perception: Memory for things observed. Perception \& Psychophysics, 64, 882-895.

KerZeL, D. (2000). Eye movements and visible persistence explain the mislocalization of the final position of a moving target. Vision Research, 40, 3703-3715.

Kowler, E., Anderson, E., Dosher, B., \& Blaser, E. (1995). The role of attention in the programming of saccades. Vision Research, 35, 1897-1916.

Legault, E., \& Standing, L. (1992). Memory for size of drawings and of photographs. Perceptual \& Motor Skills, 75, 121.

Mathews, A., \& Mackintosh, B. (2004). Take a closer look: Emotion modifies the boundary extension effect. Emotion, 4, 36-45.

Melcher, D. (2001). Persistence of visual memory for scenes: A mediumterm memory may help us to keep track of objects during visual tasks. Nature, 412, 401

MoRAn, J., \& Desimone, R. (1985). Selective attention gates visual processing in the extrastriate cortex. Science, 229, 782-784.

Pollatsek, A., Bolozky, S., Well, A. D., \& Rayner, K. (1981). Asymmetries in the perceptual span for Israeli readers. Brain \& Language, 14, 174-180.

PotTer, M. C. (1976). Short-term conceptual memory for pictures. Journal of Experimental Psychology: Human Learning \& Memory, 2, 509-522.

RENSINK, R. A. (2000). Seeing, sensing, and scrutinizing. Vision Research, 40, 1469-1487.

SANOCKI, T. (2003). Representation and perception of scenic layout. Cognitive Psychology, 47, 43-86.

Simons, D. J., \& Levin, D. T. (1997). Change blindness. Trends in Cognitive Sciences, 1, 261-267.

Tipper, S. P., Howard, L. A., \& Houghton, G. (1998). Action-based mechanisms of attention. Philosophical Transactions of the Royal Society of London: Series B, 353, 1385-1393.

Tse, P. U., Sheinberg, D. L., \& Logothetis, N. K. (2003). Attentional enhancement opposite a peripheral flash revealed using change blindness. Psychological Science, 14, 91-99.

\section{NOTE}

1. The important outcome for the purposes of the present research is that significant BE occurred on all four sides when the viewers maintained fixation. It is interesting to note that a significant left-right asymmetry in the amount of $\mathrm{BE}$ was obtained $[t(23)=2.07$; see Figure 3$]$. We thought that this might be related to the rightward bias observed in representational momentum (Halpern \& Kelly, 1993) or to the rightward bias in the perceptual reading span for English readers (Pollatsek, Bolozky, Well, \& Rayner, 1981), but the bias was not evident in the subsequent experiments reported here; nor to our knowledge, has it been reported in other BE studies.

(Manuscript received April 8, 2005; revision accepted for publication August 2, 2005.) 